\title{
Long-term sensibility outcomes of secondary digital nerve reconstruction with sural nerve autografts: a retrospective study
}

\author{
Tomasz Dębski ${ }^{1}$ [ $\cdot$ Marcin Złotorowicz² Bartłomiej Henryk Noszczyk $^{1}$
}

Received: 7 March 2021 / Accepted: 7 July 2021 / Published online: 19 July 2021

(c) The Author(s) 2021

\begin{abstract}
Background Recovery of sensibility after digital nerve injury is crucial for restoring normal hand function. We evaluated long-term outcomes of digital nerve reconstruction with autografts.

Methods This retrospective study included patients who underwent secondary reconstruction of digital nerves with nerve autografting. Recovery of sensibility was evaluated based on the following: patient self-assessment, two-point discrimination (2PD), and a total sensation score (sum of proprioception, temperature sensation, and sharp/dull discrimination). Mixed models regression was used to study predictors of sensibility outcomes. The predictors analyzed were age, sex, smoking status, number of fingers involved in a patient (as a measure of injury severity), time to reconstruction, and time to follow-up. Results In 61 patients, 174 digital nerves in 126 fingers were reconstructed after an average of 33.1 weeks from injury. The mean follow-up was 6.4 years from reconstruction. The mean graft length was $3.6 \mathrm{~cm}$. Self-rated sensibility in the affected area was very good in $13 \%$ of patients, good in 33\%, satisfactory in $40 \%$, and poor in $24 \%$. 2PD at $6 \mathrm{~mm}$ was present in $17 \%$ of patients, at $10 \mathrm{~mm}$ in $12 \%$, and at $15 \mathrm{~mm}$ in $18 \%$ (mean 2PD was 10.8). Proprioception was preserved in 107 (85\%) fingers, sensation of temperature was preserved in $99(75 \%)$ of fingers, and sharp/dull discrimination in $88(70 \%)$ fingers. Time from injury to reconstruction was the only significant predictor of the total sensation score.

Conclusion Our data indicate that earlier reconstruction is associated with a favorable outcome.
\end{abstract}

Keywords Secondary digital nerve reconstruction $\cdot$ Sural nerve autograft $\cdot$ Long-term surgical outcomes

\section{Introduction}

Traumatic hand injury is common, and it requires nerve reconstruction in about $6.1-10 \%$ of patients. The common and proper digital nerves are most frequently affected $[1,2]$.

Restoring normal sensibility is important for the recovery of hand function. Digital nerves commonly need to be surgically reconstructed, and intensive rehabilitation usually follows [1]. End-to-end coaptation and nerve grafting are the preferred methods of repairing severed nerves [1]. This method is recommended when the wound is clean, and both nerve ends are clearly visible and easily mobilized [3].

Tomasz Dębski

info@drdebski.pl

1 Department of Plastic Surgery, Orlowski Memorial Hospital, Medical Center of Postgraduate Education, Czerniakowska Street 231, Warsaw 00-416, Poland

2 Gruca Orthopaedic and Trauma Teaching Hospital, Medical Centre of Postgraduate Education, Otwock, Warsaw, Poland
Where gaps exist, direct end-to-end coaptation cannot be used, and autografts or artificial nerve conduits are required for reconstruction. Nerve autografting is the gold standard for treating this type of injury, often with the sural nerve used as the graft when multiple nerves require repair [1, 3]. The implanted donor nerve, however, degenerates and cannot directly replace the severed nerve part [4]. Instead, it creates a supportive structure for the growing axons of the injured nerve, providing a growth-permitting scaffold, including Schwann cell basal laminae, neurotrophic factors, and adhesion molecules [4]. It is, therefore, unsurprising that sensory recovery following nerve repair is generally prolonged. A review of 14 studies of digital nerve repair showed that longer follow-up after trauma was a predictor of better functional recovery [3]. Excellent sensory recovery was only seen at follow-ups longer than 6 months [3].

In the current study, we reviewed medical records of patients who had undergone secondary digital nerve reconstruction. Our aim was to assess very long-term sensory outcomes of nerve autografting and to investigate selected 
patient and injury characteristics that may affect these outcomes.

\section{Methods}

\section{Patients}

In this retrospective study, we sent invitation letters for a follow-up visit to all patients who had undergone secondary reconstruction of digital nerves with sural nerve autografting in our Department between 1994 and 2002. Primary nerve repair was not possible in these patients because of gap formation at the nerve injury site (mainly crash injury type) or unavailability of nerve grafting at the primary hospital. In all selected patients, contralateral sural nerves were used for reconstructions. Surgery was performed under regional anesthesia in a bloodless field (pneumatic cuff tourniquet) using surgical microscope (Leica, Austria). All injured nerves were dissected, both stumps were refreshed and epineural tensionless coaptation with 10-0 Prolene suture (Ethicon, USA) was performed (Fig. 1). After surgery, the hand was immobilized for 6 weeks in a dorsal blocking splint with a hand in a neutral position regardless of the site of injury. After immobilization patients received 3 months of physiotherapy. All surgeries were performed at the Plastic Surgery Department in Warsaw by surgeons with at least 3 years of training in digital nerve repair. Our hospital was, at the time of the study, a tertiary referral hospital providing services in the Mazovian district with a population of 5,356,838. As a referral hospital, we also admitted severe hand injury cases or cases after primary treatment from other districts in Poland [2]. Secondary digital nerve reconstruction with sural nerve was performed on an average of $10-15$ patients per year. In cases primarily treated in our department stabilization of bone fractures, flexor/extensor tendons repair or soft tissue coverage reconstruction was performed first. Injured digital nerves were reconstructed after 1-3 months depending on the complexity of the case.

The study protocol was approved by the Bioethical Committee of the Medical Centre of Postgraduate Education in Warsaw and conducted in accordance with the Helsinki Declaration (No. 13/PB/2012).

\section{Assessments}

We reviewed medical records of all patients included to obtain the following variables: age at reconstruction, sex, smoking status at reconstruction, graft length, primary injury mechanism, concomitant injuries to other hand structures, time from injury to reconstructive surgery, and time from surgery to follow-up. Before nerve reconstruction, all patients had absent sensibility in the area of reconstructed nerve.

During the follow-up visit, a standardized hand examination was performed as previously described [5-7]. The affected hands were examined for skin lesions, scars, fingernail abnormalities, and hypohydrosis. Patients completed a short questionnaire to assess sensibility within the fingers innervated by the reconstructed nerve as very good, good, satisfactory, or poor.

Two point discrimination (2PD) was assessed in each reconstructed nerve with a Dellon discriminator. Depending on which nerve was affected, the ulnar or radial side of the finger was touched with either one or both tips of instrument. The 2PD was scored as 1 (at $6 \mathrm{~mm}$ ), 2 (at $10 \mathrm{~mm}$ ), 3 (at 15), and 4 (>15 mm).
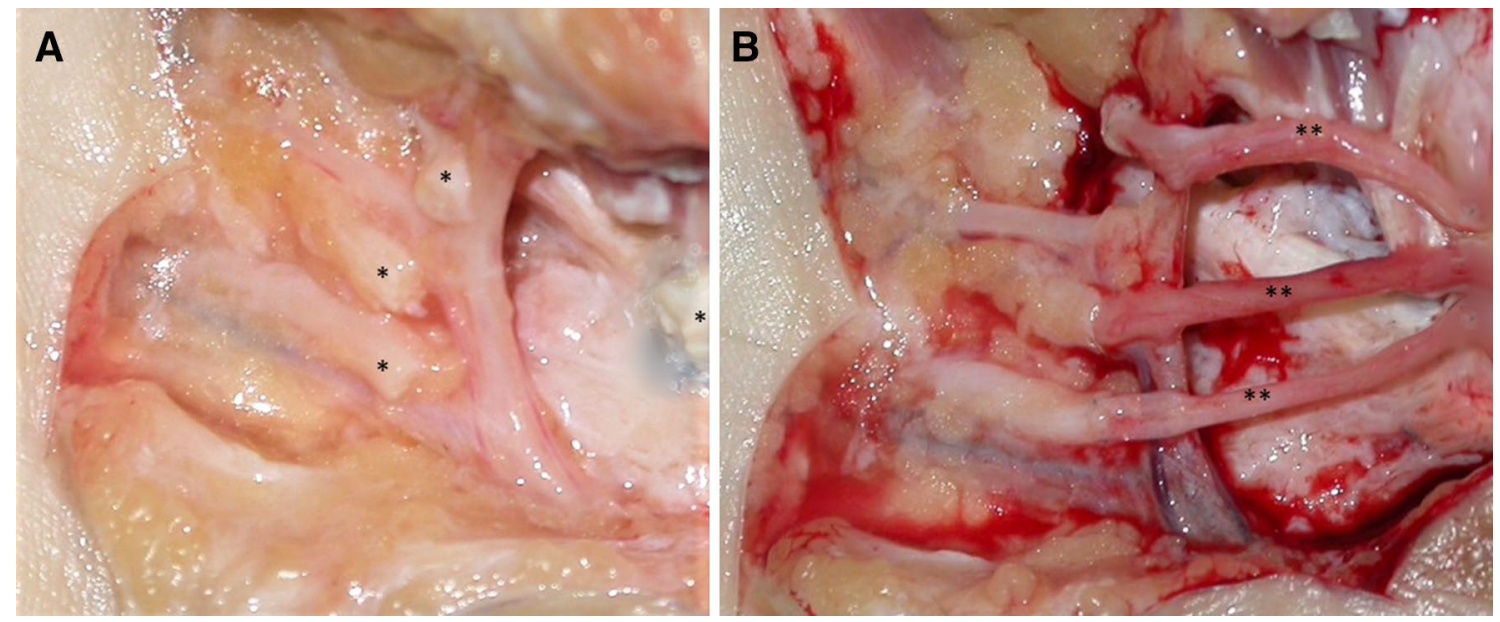

Fig. 1 Common digital nerve reconstruction with sural nerve. A A gap between proximal and distal III, III, IV common digital nerve stumps (*) after refreshing, B nerve reconstruction with sural nerve autograft $(* *)$ 
Finally, in each reconstructed nerve, we tested three aspects of sensation: proprioception, temperature sensation, and sharp/dull discrimination. Proprioception was tested by asking the patient to close their eyes and tell their finger position during passive flexion or extension. The ability to sense temperature was tested by touching the skin area innervated by the reconstructed nerve with cotton wool swabs dampened with either warm $\left(35^{\circ} \mathrm{C}\right)$ or cold water $\left(10^{\circ} \mathrm{C}\right)$. The ability to discriminate between sharp and dull was tested in a pinprick test: we touched the skin innervated by the reconstructed nerve with either the tip or the head of a sharp pin and asked the patient to tell, with their eyes closed, which end of the pin was felt (sharp or dull). All aspects of sensation were rated as either present or absent, and were added to obtain a total sensation (TS) score ( 0 , no sensation; 3 , all aspects of sensation preserved).

\section{Statistical analysis}

Descriptive statistics were calculated for all following variables: means (ranges), for continuous variables, and counts (percentages), for categorical variables. Mixed models regression was applied to study predictors of sensation after nerve reconstruction. We used data for individual nerves, with the subject as a random effect. The mean 2PD score and the TS score were used as dependent variables. The predictors (fixed effects) were age at reconstruction, sex, smoking status, number of fingers involved in a patient (as a measure of injury severity), time to reconstruction, and time to follow-up. $P<0.05$ was considered statistically significant. Statistical analyses were performed with the R software (version 3.53).

\section{Results}

\section{Patient characteristics}

Of 86 patients invited, 61 (71\%) reported for a follow-up visit. In total, they underwent 174 nerve reconstructions in 126 fingers. The mean time of follow-up was 6.4 years (2.2-10.1 years) (Fig. 2). Most patients were males (90.2\%), and the mean age at reconstruction was 33.8 years. About half of the patients were smokers (52\%). The mean time from injury to reconstruction was 33.1 weeks (5-430 weeks). Primary treatment before reconstruction was performed in our department in 22 patients (36\%). Other 39 patients (64\%) were referred from orthopedic $(77 \%)$ or neurologic departments (23\%) for secondary reconstruction. Among 174 repaired nerves, most were damaged in crush injuries $(83 \%)$ or by cuts $(16 \%)$. Additional hand structures were affected in nearly all patients (97\%). The proper palmar digital nerves were injured more frequently than the common

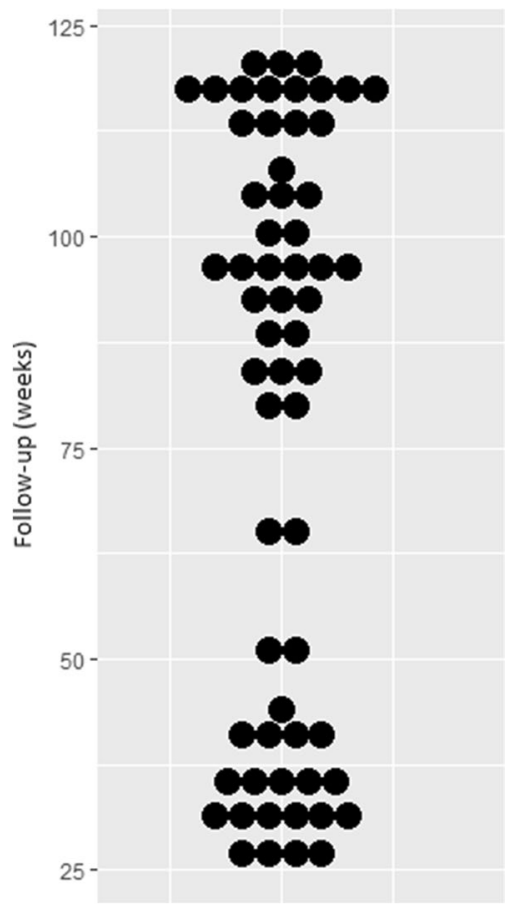

Fig. 2 Scatter plot presenting spread of follow-up times

digital nerves (64\% vs. $25 \%$ ). The mean length of sural nerve grafts was $3.6 \mathrm{~cm}$, although grafts up to $9 \mathrm{~cm}$ in length were required in some patients. The characteristics of patients at nerve reconstruction are shown in Table 1.

Self-assessed sensibility in the digits innervated by the reconstructed nerves was very good in $8(13 \%)$ patients, good in $20(33 \%)$, satisfactory in $18(30 \%)$, and poor in 39 (24\%).

\section{Assessment of hand and donor site function}

Physical examination of the digits innervated by the reconstructed nerves revealed constricting scars in 15 (25\%) of patients. The skin of the affected fingers was completely dry in $3(5 \%)$ patients, drier than in the unaffected digits in 15 (25\%), and similar as in the unaffected fingers in 43 (70\%). Complaints related to the donor site were reported by $9(15 \%)$ of patients, and they included tingling or sensory deficit in the skin area innervated by the sural nerve.

Among the 174 reconstructed nerves, the 2PD score was 1 in $30(17 \%)$ nerves, 2 in $21(12 \%)$ nerves, 3 in $31(18 \%)$ nerves, 4 in $92(53 \%)$ nerves. Among nerves with preserved 2PD $(n=82)$, the mean 2PD was $10.8 \mathrm{~mm}$. Proprioception was preserved in $148(85 \%)$ nerves, sensation of temperature was preserved in $130(75 \%)$ nerves, and sharp/dull discrimination in $122(70 \%)$ nerves. The mean ( \pm standard deviation) TS score was $2.35 \pm 1.00$. 
Table 1 Baseline patient and injury characteristics

\begin{tabular}{ll}
\hline Patient characteristics & Total $n=61$ \\
\hline Male sex, $n(\%)$ & $55(90 \%)$ \\
Age at reconstruction, mean & 33.8 years (17-63 years) \\
Smoker, $n(\%)$ & $32(52 \%)$ \\
Time from injury to reconstruction, mean & 33.1 weeks (5-430 weeks) \\
Time from reconstruction to follow-up assessment, mean & 6.4 years $(2.2-10.1$ years) \\
Dominant hand injury, $n(\%)$ & $31(51 \%)$ \\
Palmar digital nerves damaged, $n(\%)$ & \\
Common & $15(25 \%)$ \\
Proper & $39(64 \%)$ \\
Both & $7(11 \%)$ \\
Number of nerves reconstructed per patient, $n(\%)$ & \\
1 & $17(28 \%)$ \\
2 & $13(21 \%)$ \\
3 & $13(21 \%)$ \\
4 & $8(13 \%)$ \\
$\geq 5$ & $10(16 \%)$ \\
Graft length, mean (range) & $3.6 \mathrm{~cm}(1-9 \mathrm{~cm})$ \\
Isolated nerve injury, $n(\%)$ & $6(3 \%)$ \\
Primary injury type, $n(\%)$ & \\
Crush & $145(83 \%)$ \\
Cut & $28(16 \%)$ \\
Gunshot & $1(<1 \%)$ \\
Nerve reconstruction following digital replantation, $n(\%)$ & $10(6 \%)$ \\
Nerve reconstruction following digital revascularisation, $n(\%)$ & $9(5 \%)$ \\
\hline
\end{tabular}

\section{Predictors of sensation after nerve reconstruction}

Longer time from injury to reconstruction was a significant predictor of decreased TS scores, but not of $2 \mathrm{PD}$ scores at follow-up (Table 2). None of the remaining variables was significantly associated with the $2 \mathrm{PD}$ or TS scores, although the number of fingers injured was related to lower TS scores at the border of statistical significance $(P=0.054$; Tables 2, 3).

Table 2 Predictors of the TS score (proprioception, temperature, sharp/dull discrimination) at follow-up. Statistical significance $(p<0.05)$ are bolded

\section{Discussion}

In this study, we found that shorter times from injury to surgery were associated with better sensation in the longterm among patients who underwent secondary digital nerve reconstruction. This association was significant for a TS score that included proprioception, temperature sensation, and sharp-dull discrimination, but it was not significant for 2PD.

Although primary repair of peripheral nerves offers improved clinical outcomes compared to secondary reconstruction [8], it is not always feasible in patients presenting with large, contaminated, or crush wounds or when

\begin{tabular}{lrllrl}
\hline Predictor (fixed factor) & Estimate & Standard error & $\begin{array}{l}\text { Degrees of } \\
\text { freedom }\end{array}$ & $T$ value & $P$ \\
\hline Number of fingers injured & -0.236 & 0.119 & 49.650 & -1.971 & 0.054 \\
Smoking & 0.094 & 0.234 & 49.504 & 0.401 & 0.689 \\
Age at reconstruction & 0.006 & 0.009 & 50.210 & 0.669 & 0.506 \\
Graft length per nerve (cm) & 0.002 & 0.035 & 84.450 & 0.061 & 0.951 \\
Time to reconstruction (weeks) & $\mathbf{- 0 . 0 0 5}$ & $\mathbf{0 . 0 0 2}$ & $\mathbf{5 5 . 8 1 8}$ & $-\mathbf{2 . 3 7 2}$ & $\mathbf{0 . 0 2 1}$ \\
Time to follow-up (weeks) & -0.003 & 0.003 & 50.314 & -0.812 & 0.420 \\
\hline
\end{tabular}


Table 3 Predictors of 2-point discrimination at follow-up

\begin{tabular}{lrllrl}
\hline Predictor (fixed factor) & Estimate & Standard error & $\begin{array}{l}\text { Degrees of } \\
\text { freedom }\end{array}$ & $T$ value & $P$ \\
\hline Number of fingers injured & 0.106 & 0.157 & 42.759 & 0.675 & 0.503 \\
Smoking & -0.276 & 0.300 & 43.020 & -0.919 & 0.363 \\
Age at reconstruction & -0.001 & 0.012 & 45.373 & -0.117 & 0.907 \\
Graft length per nerve (cm) & 0.002 & 0.048 & 60.679 & 0.036 & 0.971 \\
Time to reconstruction (weeks) & 0.003 & 0.002 & 58.593 & 1.275 & 0.207 \\
Time to follow-up (weeks) & 0.002 & 0.004 & 44.605 & 0.644 & 0.522 \\
\hline
\end{tabular}

the neighboring hand structures are substantially affected. In these patients, secondary repair may be indicated. Our study reports very long-term (up to 10 years) outcomes of secondary digital nerve reconstruction with nerve autografts. Although, in our study, protective sensation recovered in nearly all patients, 2PD was restored only in about half of them, which suggests incomplete sensory recovery. Although 2PD is widely used to test sensory function, other measures, like the modified Highet classification [9], should perhaps be used in addition to 2PD [1]. Such measures take into account the ability to sense touch, pain or temperature, or hyperalgesia [1,9].

Paprottka et al. performed a systematic review and metaanalysis to compare outcomes of digital nerve reconstruction after different procedures, including nerve grafting [1]. In that meta-analysis, the percentage of patients with good to excellent sensory recovery ranged between 14 and $67 \%$ [1]. More recently, Stang et al. reported that, of 28 patients who received a posterior interosseous nerve graft or medial antebrachial cutaneous nerve graft, $46 \%$ achieved S4 sensibility and $36 \%$ achieved S3 + sensibility, on the modified Highet classification [10]. In our study, where a 2PD result $<15 \mathrm{~mm}$ was achieved, the modified Highet score would be $\mathrm{S} 3+(30 \%$, reconstruction cases with good and satisfactory 2PD result), or S4 (17\%, very good 2PD result). Thus, our results are similar to those of other studies on nerve grafting for digital nerve repair. The mean 2PD of $10.8 \mathrm{~mm}$, in our study, was also similar to previous reports. After a mean follow-up of 23 months, Chen et al. reported a mean 2PD score of $9.2 \mathrm{~mm}$ in 31 patients with 38 proper digital nerve defects, who underwent sural nerve grafting [11]. Another study by the same authors reported a mean 2PD score of $9.4 \mathrm{~mm}$ in 27 patients with injuries to proper digital nerves and the thumb who received sural or medial antebrachial cutaneous nerve grafts [12].

Fifteen percent of patients in our study reported problems with the donor site. Although there are sources of shorter nerve grafts in the upper extremity (cutaneous antebrachial nerve or dorsal interosseous nerve), which reduce donor site morbidity, we consider the sural nerve a useful source of long grafts required for the repair of injuries affecting multiple nerves or those associated with substantial gaps between severed nerve ends (in our study mean length of sural nerve grafts was $3.6 \mathrm{~cm}$ ). Donor site morbidity in our study compares favorably with two earlier reports, in which, after several years from sural nerve harvesting, 5-17\% of patients reported pain, 5-34\% reported cold sensitivity, $24-48 \%$ reported discomfort, tingling, or increased skin sensation, and $17 \%$ reported minimal levels of functional impairment [13, 14].

A range of commercial products, including de-cellularized nerve allografts and collagen-based or synthetic nerve guides/conduits, have been developed, and their application in peripheral nerve repair has been extensively reviewed (see for example Arslantunali, et al. Khoe et al. and Kaushik and Hammert) [15-17]. Although autologous tissues, such as muscles and/or veins, may also be used to form a nerve conduit, commercial products are available off-the-shelf and minimize the time needed for tissue harvesting and conduit preparation. These products may substitute for nerve autografts, allowing the surgeon to bridge the gap between severed ends of a nerve without the need to denervate another area and to avoid donor site morbidity. Selected prospective and registry-based studies have reported high rates of meaningful recovery ( $\mathrm{S} 3+$ or $\mathrm{S} 4$ on the modified Highet scale) with commercially available products for bridging nerve gaps: $84 \%$ with processed nerve allografts [18], 63\% with collagen nerve conduits [19], and 74\% with synthetic conduits [20]. However, despite a wealth of published literature, considerable differences between individual studies exist in terms of utilizing primary vs. secondary repair, average gap length and patient characteristics, which precludes a robust comparison of different repair methods. A large, prospective randomized study of different options for bridging digital nerve gaps, stratified by key prognostic factors, could provide information on the value of different techniques, including autografting.

In addition to describing long-term outcomes of nerve grafting, we also investigated patient- and injury-related factors that could affect sensory recovery. We found that the time from injury to reconstruction was significantly related to the total sensation (TS) score that included proprioception, temperature sensation, and sharp/dull discrimination. In contrast, we did not find any predictors of 
2PD discrimination. Our findings suggest that $2 \mathrm{PD}$ and other types of sensation may be restored differentially by digital nerve reconstruction. The sensation of temperature, for example, is a protective sensation, which forms an earlier step towards full sensory recovery according to the modified Highet classification [9] than fine, discriminative touch examined with the 2PD test. Moreover, no other predictors of sensibility after nerve reconstruction in our study proved significant, including smoking. This observation contrasts with some previous studies [21-24]. Similarly, younger age at reconstruction [3, 24, 25], shorter grafts [25], and isolated nerve injury [24] have all been found to be associated with favorable sensory outcomes after nerve reconstruction within the upper limb. The follow-up time in our study was also not related to sensory outcomes, which is in line with the report by Bulut et al. [24], but does not agree with the observations reported by Meermans et al. [3]. Although graft length was not found to be significantly associated with sensory recovery in our study, previous studies reported an association between sensory recovery and the length of the gap between severed nerve ends. He et al. found shorter gap length to be an independent predictor of good to excellent sensory recovery after the repair of both purely sensory and mixed nerves [25]. The review by Mermans et al. identified a tendency (not statistically significant) for worse sensory recovery with increasing length of gap [3]. Female sex has also been associated with significantly better sensory recovery compared with males, likely due to lower injury severity in women, who usually perform only light manual labor, or due to women showing better compliance with postoperative treatment [25]. As women constituted only a tenth of the patients in our study, this sex imbalance could have affected our results. Finally, a recent study by Fakin et al., investigating sensory outcomes following end-toend coaptation of 93 digital nerves [26], found surgeon experience to be the only significant predictor of sensory outcome when accounted for age, smoking, mechanism of injury, lesions or anastomosis of digital artery, or time of immobilization [26]. This finding highlights the importance of treating patients with digital nerve injuries at specialized centers with teams experienced in repairing small peripheral nerves. Our study was conducted at a specialist reconstructive surgery center, and all surgeons had at least 3 years of training in digital nerve repair.

Our study was limited because it was observational, retrospective, and single-center. Of 86 patients, only 61 (71\%) reported for follow-up, which could lead to bias. Moreover, we did not evaluate surgical outcomes with regards to activities of daily live. Furthermore, the effect of surgeon experience on sensory outcome was not assessed. The strengths of our study include a relatively large sample size and a long follow up.

\section{Conclusions}

This study presents long-term outcomes of secondary digital nerve repair with nerve autografts, which might be valuable to patients and clinicians. Although three quarters of patients rated reconstruction outcomes as at least satisfactory, 2PD was poor in approximately half of the patients. Only the time from injury to reconstruction was a significant predictor of sensibility (proprioception, temperature sensation, sharp/ dull discrimination). We did not find any significant predictors of 2PD.

Author contributions TD wrote an article, designed and performed the study, MZ performed the study, BN supervised the study.

Funding No funding.

\section{Declarations}

Conflict of interest The authors declare no conflict of interest.

Ethics approval Bioethical Committee of the Medical Centre of Postgraduate Education in Warsaw and conducted in accordance with the Helsinki Declaration (No. 13/PB/2012).

Open Access This article is licensed under a Creative Commons Attribution 4.0 International License, which permits use, sharing, adaptation, distribution and reproduction in any medium or format, as long as you give appropriate credit to the original author(s) and the source, provide a link to the Creative Commons licence, and indicate if changes were made. The images or other third party material in this article are included in the article's Creative Commons licence, unless indicated otherwise in a credit line to the material. If material is not included in the article's Creative Commons licence and your intended use is not permitted by statutory regulation or exceeds the permitted use, you will need to obtain permission directly from the copyright holder. To view a copy of this licence, visit http://creativecommons.org/licenses/by/4.0/.

\section{References}

1. Paprottka FJ, Wolf P, Harder Y, et al. Sensory recovery outcome after digital nerve repair in relation to different reconstructive techniques: meta-analysis and systematic review. Plast Surg Int. 2013;2013:704589.

2. Dębski T, Noszczyk BH. Epidemiology of complex hand injuries treated in the Plastic Surgery Department of a tertiary referral hospital in Warsaw. Eur J Trauma Emerg Surg. 2020. https://doi. org/10.1007/s00068-020-01312-5

3. Mermans JF, Franssen BBGM, Serroyen J, Van der Hulst RRWJ. Digital nerve injuries: a review of predictors of sensory recovery after microsurgical digital nerve repair. Hand. 2012;7(3):233-41.

4. Grinsell D, Keating CP. Peripheral nerve reconstruction after injury: a review of clinical and experimental therapies. Biomed Res Int. 2014;2014:698256.

5. Jerosch-Herold C. A study of the relative responsiveness of five sensibility tests for assessment of recovery after median nerve injury and repair. J Hand Surg Br. 2003;28(3):255-60. 
6. Wong KH, Coert JH, Robinson PH, Meek MF. Comparison of assessment tools to score recovery of function after repair of traumatic lesions of the median nerve. Scand J Plast Reconstr Surg Hand Surg. 2006;40(4):219-24.

7. Rosén B. Recovery of sensory and motor function after nerve repair. A rationale for evaluation. J Hand Ther. 1996;9(4):315-27.

8. Zeineldin AA, Mohammed MAS, Elhoseny AAL. Comparative study between primary versus delayed peripheral nerve repair after various types of injury. Menoufia Med J. 2015;28(1):80-6.

9. Mackinnon SE, Dellon AL. Surgery of the peripheral nerve. New York: Thieme Medical Publishers; 1988.

10. Stang F, Stollwerck P, Prommersberger KJ, van Schoonhoven J. Posterior interosseus nerve vs. medial cutaneous nerve of the forearm: differences in digital nerve reconstruction. Arch Orthop Trauma Surg. 2013;133(6):875-80.

11. Chen C, Tang P, Zhang X. Finger sensory reconstruction with transfer of the proper digital nerve dorsal branch. J Hand Surg Am. 2013;38(1):82-9.

12. Chen $C$, Tang P, Zhang X. Reconstruction of proper digital nerve defects in the thumb using a pedicle nerve graft. Plast Reconstr Surg. 2012;130(5):1089-97.

13. Ijpma FF, Nicolai JP, Meek MF. Sural nerve donor-site morbidity: thirty-four years of follow-up. Ann Plast Surg. 2006;57(4):391-5.

14. Hallgren A, Björkman A, Chemnitz A, Dahlin LB. Subjective outcome related to donor site morbidity after sural nerve graft harvesting: a survey in 41 patients. BMC Surg. 2013;24(13):39.

15. Arslantunali D, Dursun T, Yucel D, Hasirci N, Hasirci V. Peripheral nerve conduits: technology update. Med Devices. 2014;7:405-24.

16. Kehoe S, Zhang XF, Boyd D. FDA approved guidance conduits and wraps for peripheral nerve injury: a review of materials and efficacy. Injury. 2012;43(5):553-72.

17. Kaushik AP, Hammert WC. Options for digital nerve gap. J Hand Surg Am. 2015;40(1):141-4.
18. Rinker BD, Ingari JV, Greenberg JA, Thayer WP, Safa B, Buncke GM. Outcomes of short-gap sensory nerve injuries reconstructed with processed nerve allografts from a multicenter registry study. J Reconstr Microsurg. 2015;31(5):384-90.

19. Lohmeyer JA, Kern Y, Schmauss D, et al. Prospective clinical study on digital nerve repair with collagen nerve conduits and review of literature. J Reconstr Microsurg. 2014;30(4):227-34.

20. Weber RA, Breidenbach WC, Brown RE, Jabaley ME, Mass DP. A randomized prospective study of polyglycolic acid conduits for digital nerve reconstruction in humans. Plast Reconstr Surg. 2000;106(5):1036-45 (discussion 1046-8).

21. Rinker B, Liau JY. A prospective randomized study comparing woven polyglycolic acid and autogenous vein conduits for reconstruction of digital nerve gaps. J Hand Surg Am. 2011;36(5):775-81.

22. Al-Ghazal SK, McKiernan M, Khan K, McCann J. Results of clinical assessment after primary digital nerve repair. J Hand Surg Br. 1994;19(2):255-7.

23. Safa B, Rinker B, Weber RV, et al. Comparisons of patient smoking status and functional recovery following peripheral nerve repair with processed nerve allograft. In: Paper presented at: 2015 American Association for Hand Surgery Meeting. January 21-24, 2015, Paradise Island, Bahamas. http://meeting.handsurgery.org/ abstracts/2015/95.cgi. Accessed June 2017.

24. Bulut T, Akgün U, Çıtlak A, Aslan C, Şener U, Şener M. Prognostic factors in sensory recovery after digital nerve repair. Acta Orthop Traumatol Turc. 2016;50(2):157-61.

25. He B, Zhu Z, Zhu Q, et al. Factors predicting sensory and motor recovery after the repair of upper limb peripheral nerve injuries. Neural Regen Res. 2014;9(6):661-72.

26. Fakin RM, Calcagni M, Klein HJ, Giovanoli P. Long-term clinical outcome after epineural coaptation of digital nerves. J Hand Surg Eur. 2016;41(2):148-54. 